\title{
Effects of Additional Oxygen Flow on the Optical and Electrical Properties of Ion Beam Sputtering Deposited Molybdenum-Doped Zinc Oxide Layer
}

\author{
Chin-Chiuan Kuo, ${ }^{1}$ Chi-Chang Liu, ${ }^{1}$ Shao-Chih He, ${ }^{2}$ Jing-Tang Chang, ${ }^{3}$ and Ju-Liang He ${ }^{2}$ \\ ${ }^{1}$ Department of Mechanical Engineering, Chien Kuo Technology University, Changhua City 500, Taiwan \\ ${ }^{2}$ Department of Materials Science and Engineering, Feng Chia University, Taichung City 40724, Taiwan \\ ${ }^{3}$ Graduate Institute of Biomedical Materials and Tissue Engineering, College of Oral Medicine, Taipei Medical University, \\ Taipei City 110, Taiwan
}

Correspondence should be addressed to Chin-Chiuan Kuo, cckuo@ctu.edu.tw

Received 20 March 2012; Revised 4 May 2012; Accepted 6 May 2012

Academic Editor: Ping Xiao

Copyright ( $\odot 2012$ Chin-Chiuan Kuo et al. This is an open access article distributed under the Creative Commons Attribution License, which permits unrestricted use, distribution, and reproduction in any medium, provided the original work is properly cited.

\begin{abstract}
The transparent conductive molybdenum-doped zinc oxide (MZO) was deposited onto a flexible polyethersulfone (PES) substrate by using an ion beam sputtering system. An argon ion beam was used to sputter an MZO target at constant pressure of $0.67 \mathrm{~Pa}$ and substrate temperature of $130^{\circ} \mathrm{C}$ with varying the oxygen flow rate from 0 to $12 \mathrm{sccm}$. The influences of additional oxygen flow on the microstructure, optical, and electrical properties of films were investigated. The obtained MZO films present a crystalline structure. With increasing the oxygen flow rate, their electrical resistivity increases, and the optical band gap decreases from 3.46 to $3.20 \mathrm{eV}$. The film deposited in the atmosphere without introducing oxygen exhibits the best optical transmittance of $82.9 \%$ at $550 \mathrm{~nm}$ wavelength, electrical resistivity of $8.32 \times 10^{-3} \Omega \mathrm{cm}$, carrier concentration of $6.82 \times 10^{20} \mathrm{~cm}^{-3}$, and carrier mobility of $2.45 \mathrm{~cm}^{2} / \mathrm{Vs}$.
\end{abstract}

\section{Introduction}

Transparent conductive oxide (TCO) thin films have become indispensable electrode materials in current optoelectronic products [1-4]. The most applied material system nowadays is indium tin oxide (ITO) [5]. However, the rareness and environmental hazard of indium are forcing us to search new alternative materials. A qualified TCO material must exhibit low film resistivity about $10^{-4} \Omega \mathrm{cm}$ and visible light transmittance of $80 \%$. Over all candidates, zinc oxide shows industrial advantages on low cost, low depositing temperature, and being easy to be etched and inner to hydrogen plasma $[6,7]$. The electrical conductivity of zinc oxide depends on the free carrier concentration contributed by oxygen vacancies or interstitial metal atoms in it [8]. Thus, to form a zinc oxide-based TCO semiconductor structure by doping the group 13 and 14, such as aluminum, gallium, or boron, is a well-known method for improving the conductivity of zinc oxide [9]. Substitution of Mo atoms for $\mathrm{Zn}$ sites in zinc oxide can originate extrinsic carriers, which makes the molybdenum-doped zinc oxide (MZO) the most potential one of these materials.

In comparison with the conventional glass substrates, transparent polymer substrates can be more ductile, flexible, light weighted, and formable, which make them the best chosen TCO-deposited substrates for flexible electronics, currently. However, their low thermal tolerance restricts the process temperature of other integrated materials. Various low temperature deposition techniques for TCO layers have been developed, such as pulsed laser deposition (PLD) [10], high power impulse magnetron sputtering (HiPIMS) [11], facing targets sputtering deposition (FTS) [9], ion beam assisted deposition (IBAD) [12], ion beam sputtering deposition (IBSD) $[13,14]$, and dual ion beam sputtering deposition (DIBSD) [15]. In an ion beam sputter system, an argon ion beam generated from the ion source is 


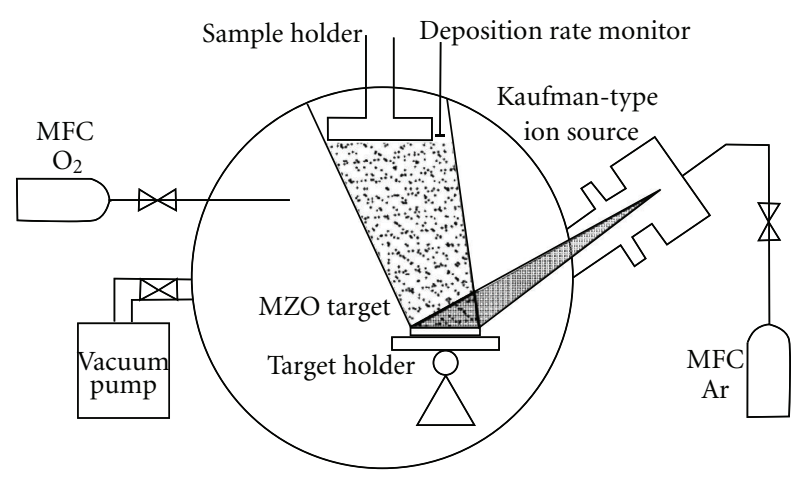

Figure 1: The schematic diagram of dual ion beam sputtering deposition system used in this study.

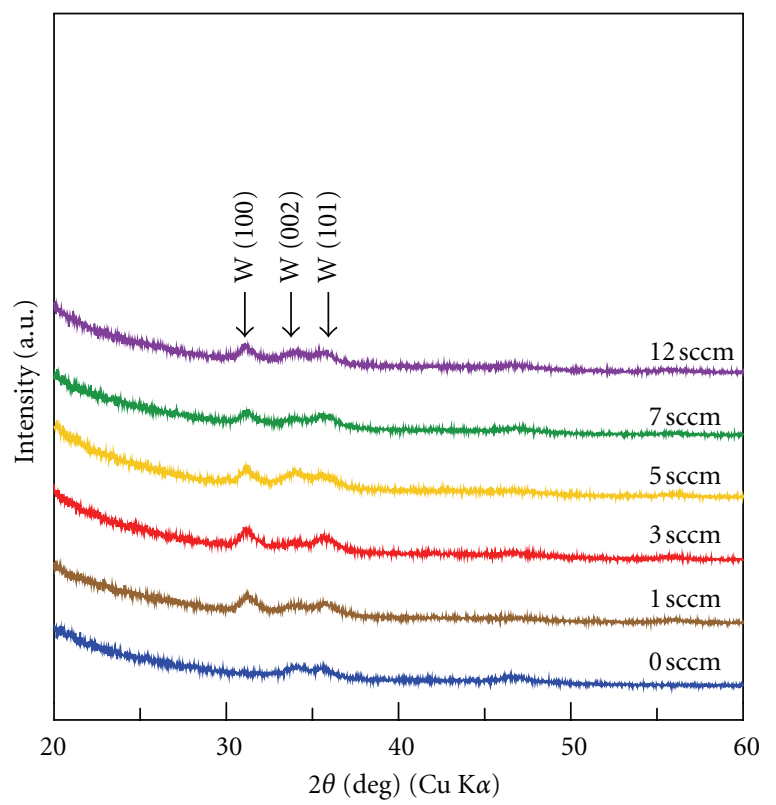

Figure 2: The X-ray diffraction patterns of MZO films deposited on $\mathrm{SiO}_{2}$-precoated PES at different oxygen flow rates.

used to sputter atoms on target surface. The high kinetic energy transferred from the incident argon species to the sputtered target material species provides adatoms the required activation energy for the nucleation and grain growth of films. It enables the deposition of crystalline MZO films onto the polyethersulfone (PES) substrates at a low processing temperature. In this research, the influences of additional oxygen flow rate on the film crystallinity, surface morphology, and optical transparency and resistivity are studied.

\section{Experimental Procedures}

A thickness of $1 \mathrm{~mm}$ thick PES sheet was cut into $2 \mathrm{~cm} \times 2 \mathrm{~cm}$ squares as substrates. The source MZO target within a size of $10.2 \mathrm{~mm}$ in diameter and $3.0 \mathrm{~mm}$ in thickness was made by LTS Chemical Inc. It composed $97 \mathrm{wt} . \% \mathrm{ZnO}$ and $3 \mathrm{wt} . \%$ $\mathrm{MoO}_{3}$ with a $4 \mathrm{~N}$ purity. The deposition of $\mathrm{MZO}$ was carried
TABLE 1: The deposition parameters for $\mathrm{SiO}_{2}$ buffer layer.

\begin{tabular}{lc}
\hline Parameter & Composition, setting \\
\hline Target material & $\mathrm{SiO}_{2}$ \\
Working pressure $(\mathrm{Pa})$ & 0.67 \\
Substrate temperature $\left({ }^{\circ} \mathrm{C}\right)$ & 130 \\
Film growth rate $(\mathrm{nm} / \mathrm{s})$ & 0.05 \\
Film thickness $(\mathrm{nm})$ & 70 \\
\hline
\end{tabular}

TABle 2: The conditions and oxygen flow settings for the MZO deposition.

\begin{tabular}{lc}
\hline Parameter & Composition, setting \\
\hline Target material & $\mathrm{ZnO} / \mathrm{MoO}_{3}(97 / 3 \mathrm{wt} \%)$ \\
Working pressure $(\mathrm{Pa})$ & 0.5 \\
Substrate temperature $\left({ }^{\circ} \mathrm{C}\right)$ & 130 \\
Film growth rate $(\mathrm{nm} / \mathrm{s})$ & 0.05 \\
Oxygen flow rate $(\mathrm{sccm})$ & $0,1,3,5,7,12$ \\
Film thickness $(\mathrm{nm})$ & 140 \\
\hline
\end{tabular}

out in a customized IBSD system (JUNSUN TECH IBS-600). The schematic diagram of this coating system is shown in Figure 1. In this system, one Kaufman-type convergent argon ion source (Veeco 05DC-PKG ASSY) with a plasma bridge neutralizer (PBN) is used for sputtering the MZO target. Prior to the film deposition, substrates were ion bombarded for $10 \mathrm{~min}$ to decontaminate and to flat the PES surface by using one KRI EH-200 end-Hall-type divergent argon ion source with a hollow cathode electron source neutralizer. After vacuuming the deposition chamber to the pressure of $1.3 \times 10^{-5} \mathrm{~Pa}$, argon gas with a constant flow rate was inlet, and a constant setting pressure of value was controlled by automatic vacuum valve.

For isolating the water or polymer vapor transmission from PES substrate into following MZO film, a single thin $\mathrm{SiO}_{2}$ layer was first coated without applying assistant ion beam. The deposition conditions of $\mathrm{SiO}_{2}$ layer are listed in Table 1. This $\mathrm{SiO}_{2}$ layer could also buffer the compressive stress in MZO and the consequent rumpling due to the substrate shrinkage. The sequent MZO depositions were carried out, respectively, under the conditions listed in Table 2. Oxygen at various flow rates between 0 to $12 \mathrm{sccm}$ was admitted individually for studying the influences of additional oxygen. The beam energy for sputtering argon ion source was set at $700 \mathrm{eV}$ with a beam current of $50 \mathrm{~mA}$ for obtaining a film growth rate of $0.05 \mathrm{~nm} / \mathrm{s}$.

The film crystal structure is analyzed by using a Bruker D8-SSS multipurpose thin-film X-ray diffractometer (XRD), at a grancing incident angle of $1.8^{\circ}$ with a scan rate of $3^{\circ} / \mathrm{min}$ from 2-theta $20^{\circ}$ to $60^{\circ}$. A HITACHI S- 4800 field-emission scanning electron microscope (FESEM) was used to observe the surface and the cross-sectional morphology of films. The composition and the bonding of deposits were analyzed by using ULVAC-PHI Electron Spectroscopy for Chemical Analysis (ESCA) with $\mathrm{Al}\left(\mathrm{K}_{\alpha}\right) \mathrm{X}$-ray. The spectra were collected after removing a $20 \mathrm{~nm}$ in deepth contaminated surface layer via the accessory argon ion beam. The electrical resistivity of 

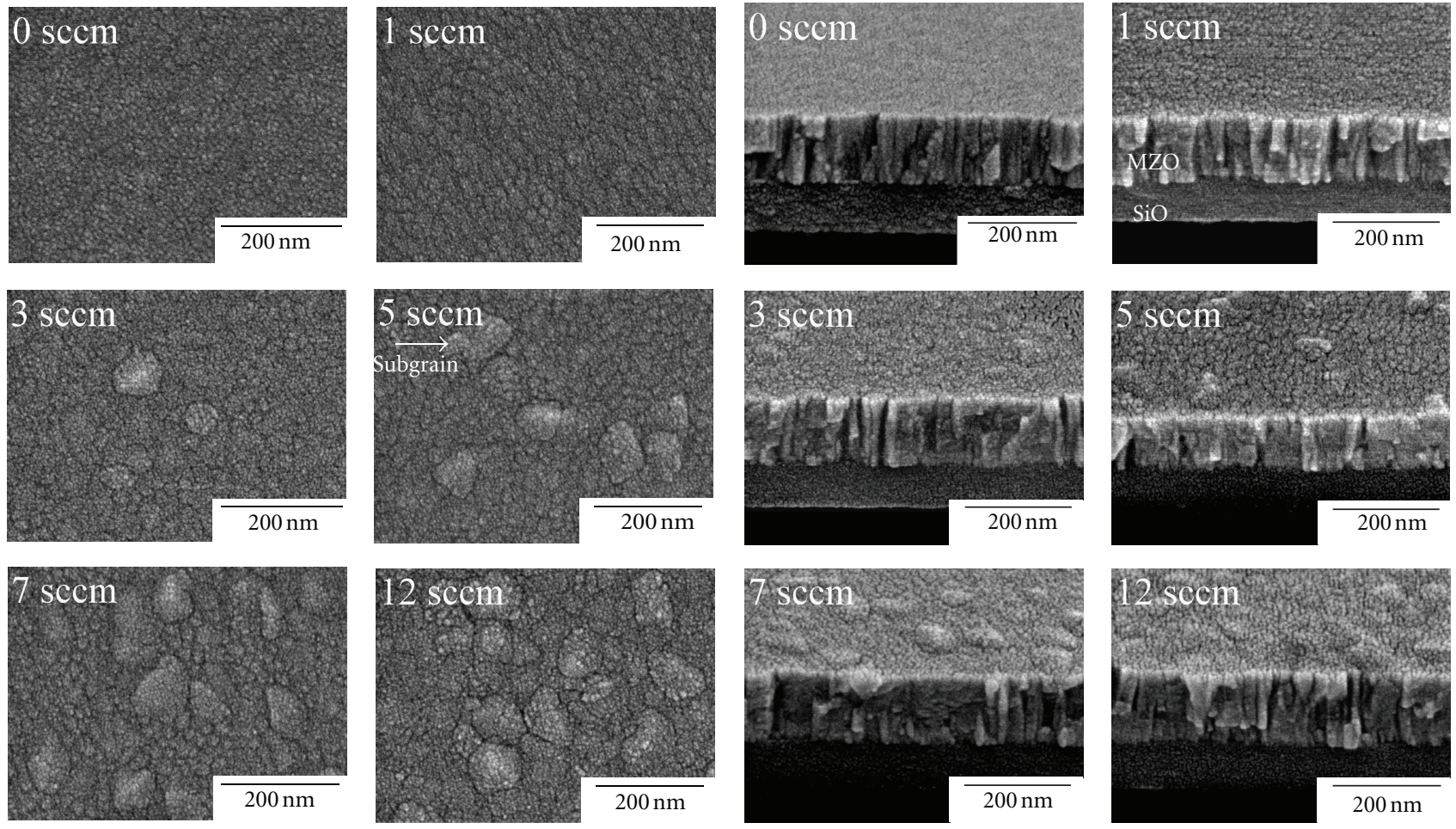

(a)

(b)

Figure 3: The (a) surface and (b) cross-sectional SEM images of MZO films deposited on $\mathrm{SiO}_{2}$-precoated PES at different oxygen flow rates.

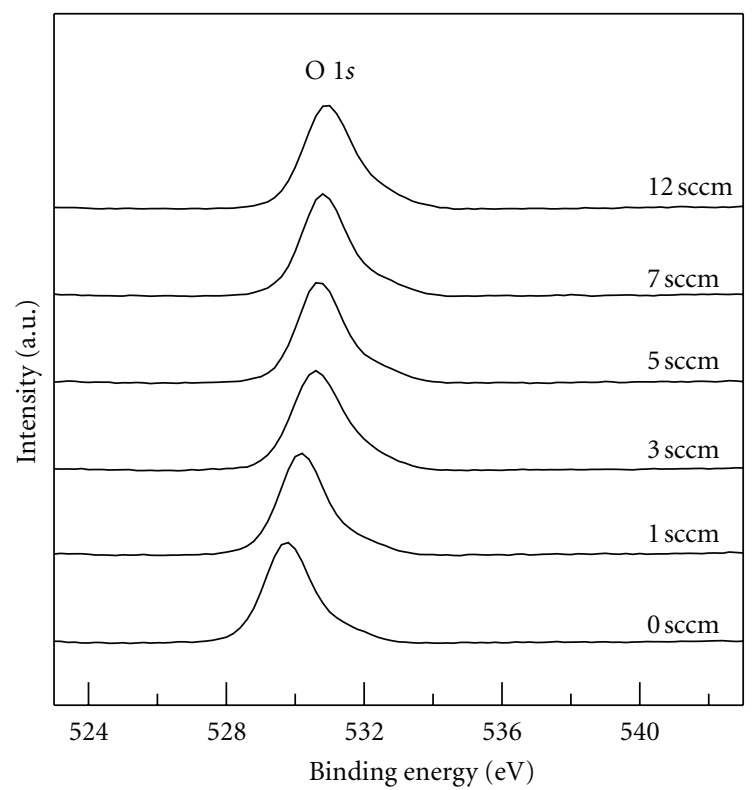

(a)

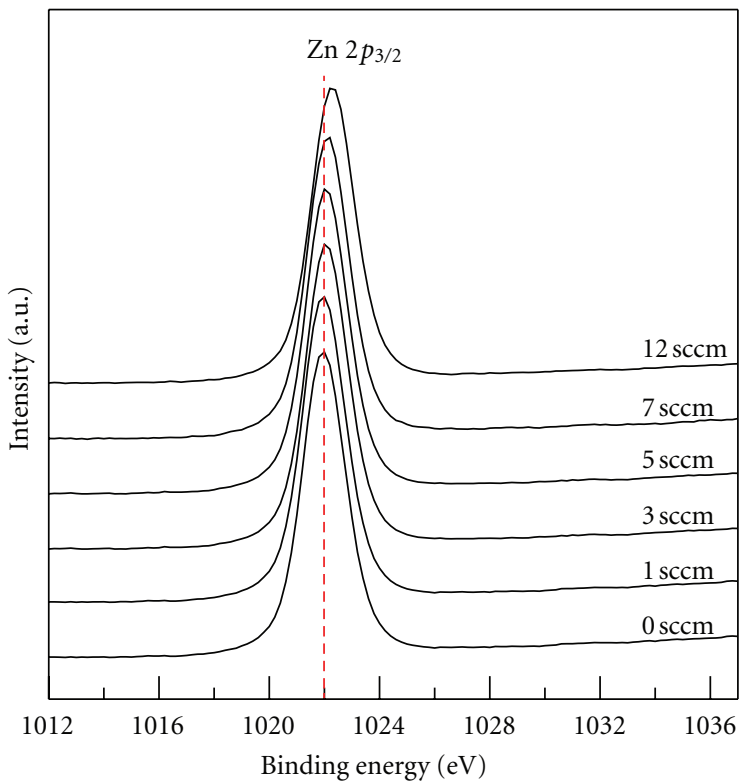

(b)

FIgUre 4: The O 1s and Zn 2p $p_{3 / 2}$ peaks in ESCA spectra of MZO films deposited at different oxygen flow rates.

MZO was measured by ultizing the Quatek QT-50 four-point probe. The carrier concentration and the carrier mobility of MZO were determined by the Hall effect measurement (Bridge Technology HMS-3000). The optical absorption and transmittance between 300 to $800 \mathrm{~nm}$ of MZOdeposited specimens were measured by a Shimadzu UV1700 ultraviolet-visible spectrophotometer and the obtained spectra were used to estimate the optical band gap $E_{g}$ values 


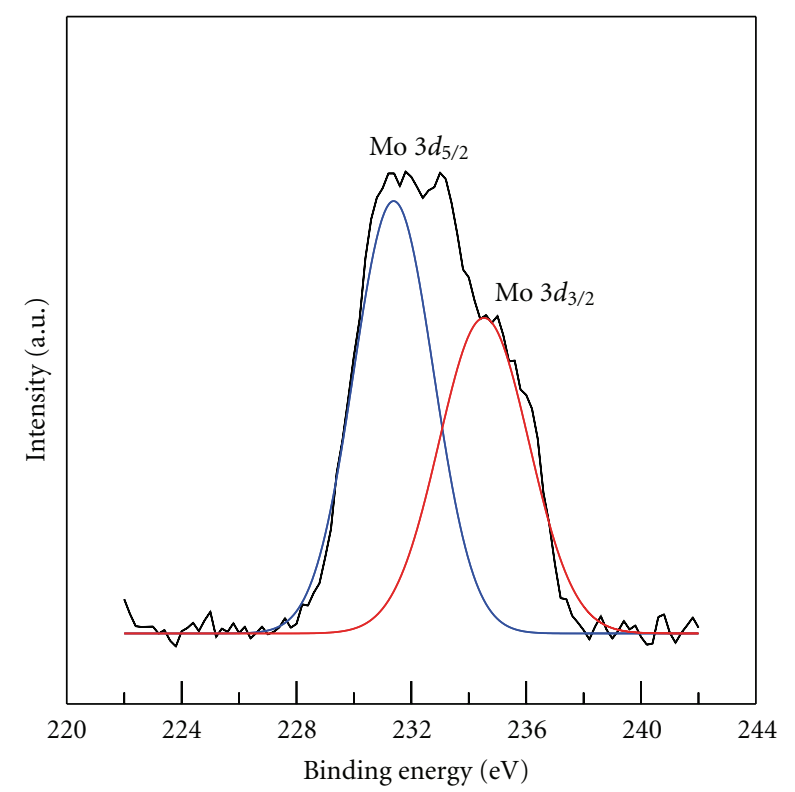

FIgure 5: The ESCA Mo $3 d$ peak of MZO film deposited without introducing oxygen flow.

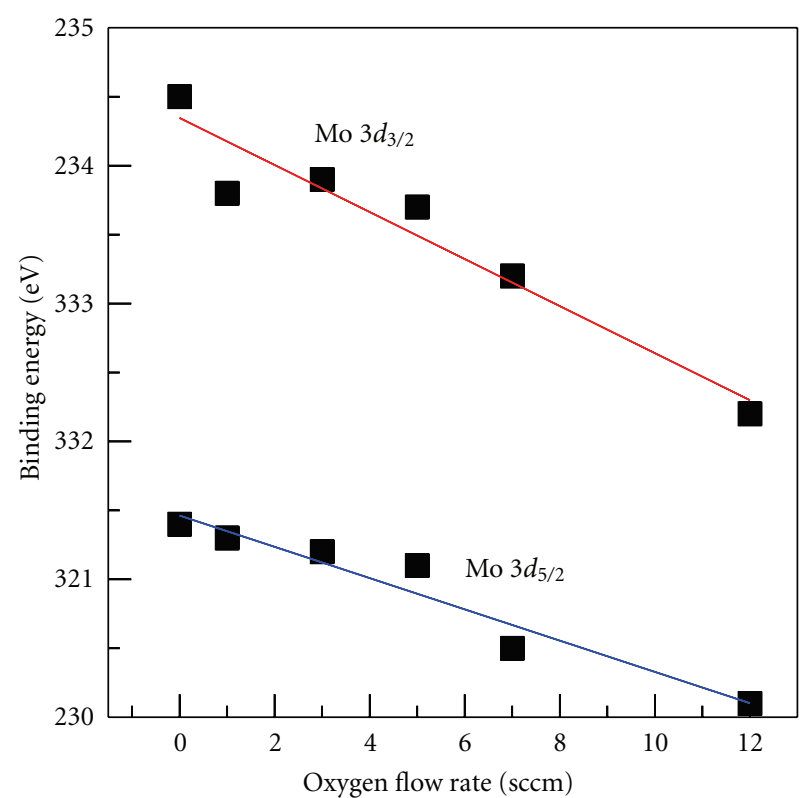

FIGURE 6: The correlation between estimated binding energy of ESCA Mo $3 d$ peaks of MZO films and deposition oxygen flow rate.

by following dependence on absorbency coefficient $\alpha$

$$
\alpha h v=A\left(h v-E_{g}\right)^{m}
$$

where $h v$ is the incident photon energy. $A$ is a constant and $m=1 / 2$ and 2 for direct and indirect band gap energy, respectively. The plots of $(\alpha h v)^{2}$ and $(\alpha h v)^{1 / 2}$ versus photon energy were compared. The graphs of $(\alpha h v)^{2}$ versus $h v$ were found to be more straight lines over the whole optical absorption spectra, which indicates a direct rather

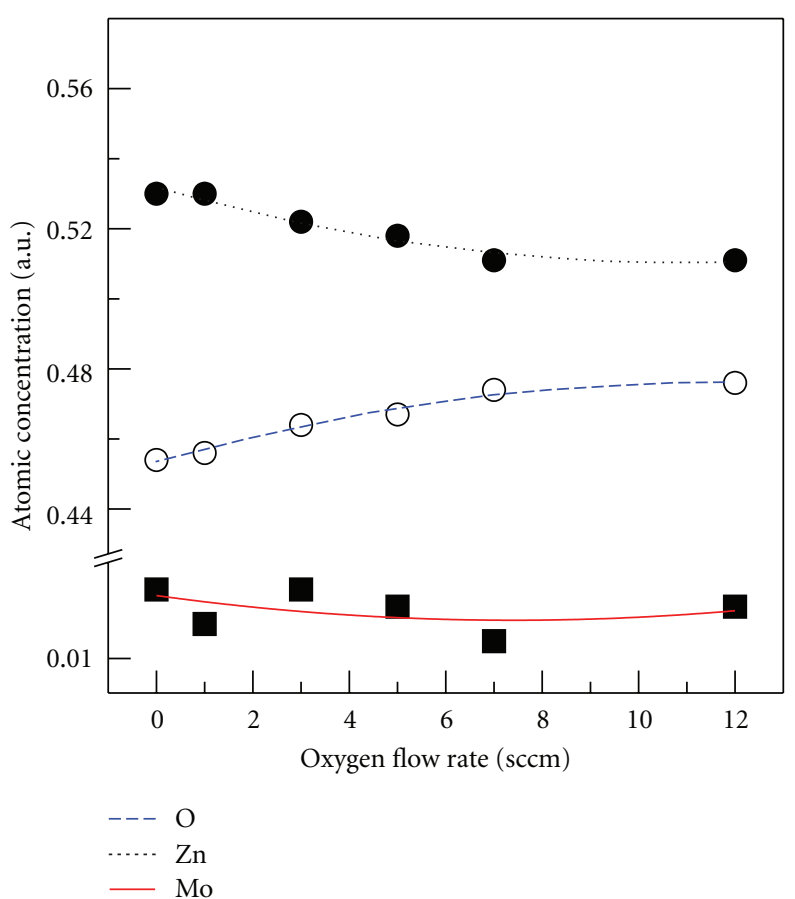

FIGURE 7: The dependence of element fractions in MZO film on deposition oxygen flow rate.

than an indirect band gap for MZO films in this study. By extrapolation of the $(\alpha h v)^{2}$ value to zero, the intercept at photo energy can be obtained as the optical band gap of the deposited MZO. The figure of merit (FOM) at different wavelengths, $T^{10} / R_{s}$, where 10 is the most favorable order of magnitude to transmissions of 0.90 [16], for each specimen is calculated from measured optical transmittance, $T$, and sheet resistance, $R_{s}$.

\section{Results and Discussion}

The XRD patterns of MZO-deposited specimens are shown in Figure 2. There are only diffraction peaks of hexagonal wurtzite phase $\mathrm{ZnO}$, labeled as $\mathrm{W}(100), \mathrm{W}(002)$, and $\mathrm{W}(101)$, and no diffraction of other compound phase is found. This demonstrates that the deposited MZO presents a polycrystalline wurtzite structure. It concurred with the Qu's work of $\mathrm{ZnO}$ film deposited in utilizing a similar technique [17]. The crystal orientations of MZO change with the oxygen flow rate. This trend was also found in $\mathrm{RF}$ magnetron sputter deposition of aluminum-doped zinc oxide (AZO) by Tsuji and Hirohashi [18]. Unlike the MZO film deposited without admitting oxygen flow, all other films deposited with introducing oxygen gas exhibit a significant diffraction peak of W(100). Introducing additional oxygen gas increases the probability of reactions between oxygen, zinc, and molybdenum species, which reduces the vacancies in the lattice, changes the lattice constants, and increases internal stress state. Consequently, the orientation of W(100) in films forms. 


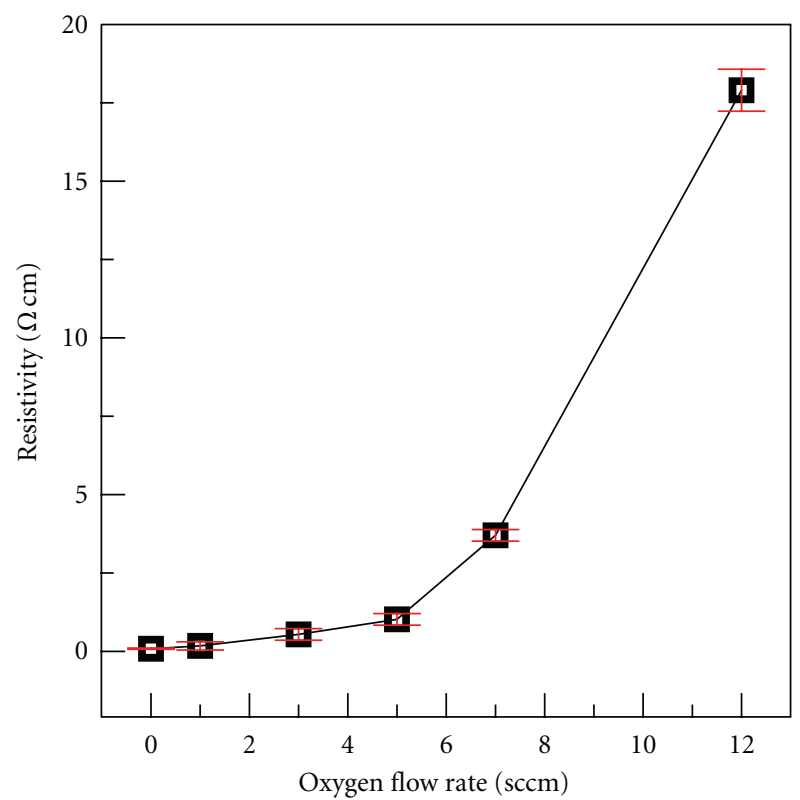

(a)

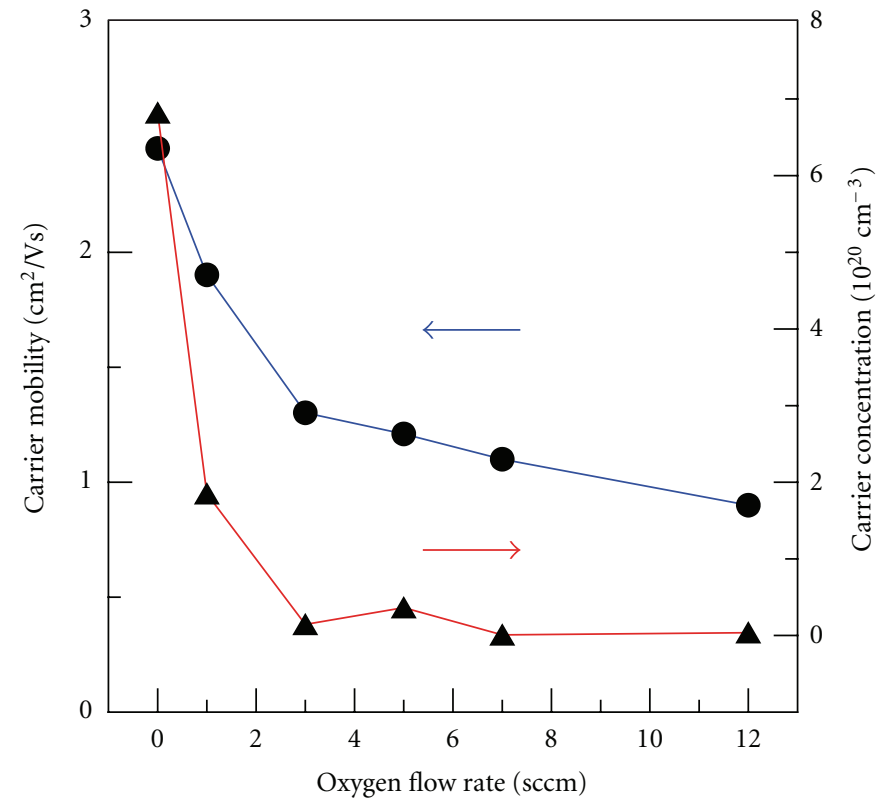

(b)

FIgURE 8: The dependence of the electrical resistivity, the carrier mobility, and the carrier concentration of MZO films on the deposition oxygen flow rate.
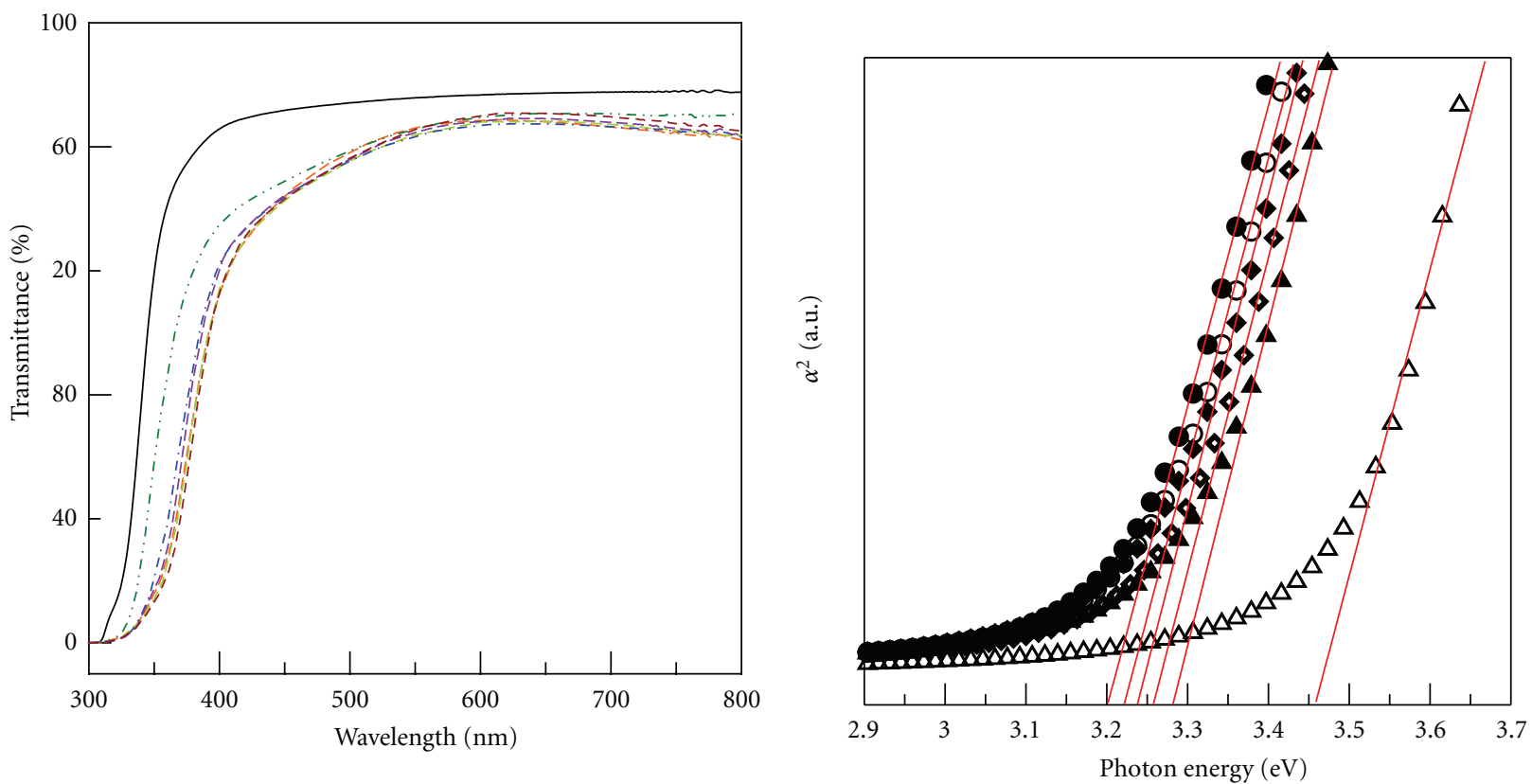

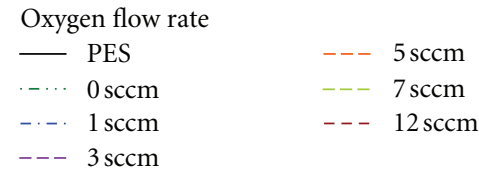

(a)
Oxygen flow rate

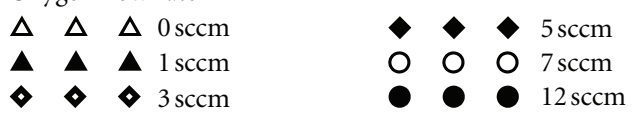

(b)

FIGURE 9: The (a) transmittance spectra and (b) estimated optical bandgap of MZO-coated PES deposited at different oxygen flow rates. 


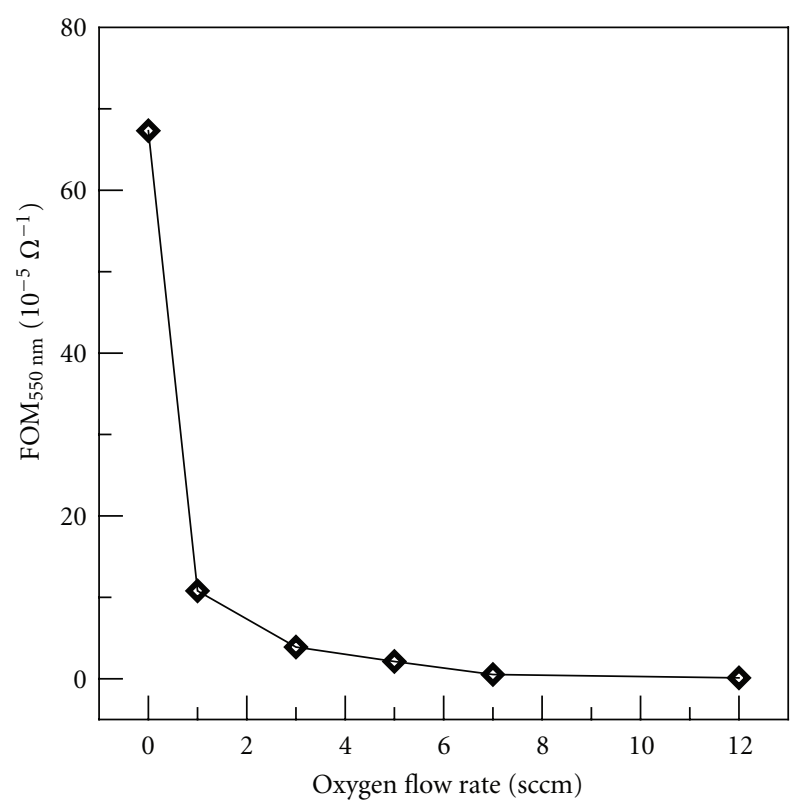

FIgure 10: The figure of merit value of MZO-coated PES prepared at different oxygen flow rates.

The surface and cross-sectional morphologies of MZO films deposited at different oxygen flow rates are shown in Figure 3. The surface projections, also called domains [19], and columnar grains both widen with oxygen flow rate, which reflects the above-mentioned orientation change. Such orientation alteration was also reported in RF magnetron sputter deposition of ITO by $\mathrm{Wu}$ and Chiou [19]. In the ion beam assisted evaporation of ITO, the surface projections also widen with the oxygen ion beam energy [20]. Both researches indicated that the excess oxygen in lattice increases the internal stress of the film. The cumulated stress drives the grain growth orientation in films to polygonization and subgrain boundary.

The $\mathrm{O} 1 s$ and $\mathrm{Zn} 2 p_{3 / 2}$ peaks in ECSA spectra of MZO films deposited at different oxygen flow rates are shown in Figure 4. The $\mathrm{O} 1 \mathrm{~s}$ peaks usually exhibit between 530.0 to $531.6 \mathrm{eV}$. The bonding with lower energy, near $530.0 \mathrm{eV}$, belongs to lattice $\mathrm{O}^{2-}$ in hexagonal wurtzite, and the bonding near $531.6 \mathrm{eV}$ could be contributed by the absorbed oxygen or oxygen-contained molecular on film surface or in defects [21]. The $\mathrm{O}$ 1s peak in $\mathrm{MZO}$ shifts from lower to higher energy level, which implies that the added oxygen flow rate during the deposition increases the amount of absorbed oxygen on film surface or trapped oxygen in defects. Excess absorption of oxygen in the film reduces the concentration of oxygen vacancy and consequent conductivity [22]. The $\mathrm{Zn}$ $2 p_{3 / 2}$ bonding energy slightly shifts from 1022.0 to $1022.2 \mathrm{eV}$. Without additional oxygen, most of zinc remains a $\mathrm{Zn}^{2+}$ state in the oxygen vacancy rich $\mathrm{ZnO}_{1-x}$ film, although the addition of oxygen makes a more stoichiometric $\mathrm{ZnO}$.

Figure 5 shows the ESCA Mo $3 d$ peak of MZO film deposited without introducing oxygen. This peak can be decomposed into two components, Mo $3 d_{5 / 2}(231.4 \mathrm{eV})$ and Mo $3 d_{3 / 2}(234.5 \mathrm{eV})$. It reveals that the molybdenum in $\mathrm{MZO}$ presents the $\mathrm{Mo}^{6+}$ state, which concurs with the Meng's results [23]. However, as shown in Figure 6, both the estimated binding energies of Mo $3 d_{5 / 2}$ and Mo $3 d_{3 / 2}$ reduce with the oxygen flow rate. It could be due to the increased binding of $\mathrm{Mo}^{6+}$ to excess oxygen; the $\mathrm{Mo}^{6+}$ acquires a valence electron from outer shell of $\mathrm{O}^{2-}$ and turns into $\mathrm{Mo}^{5+}$ state, which causes the downward shift of Mo $3 d$ [24]. The fractions of $\mathrm{O}, \mathrm{Zn}$, and $\mathrm{Mo}$ in $\mathrm{MZO}$ film deposited at different oxygen flow rates are shown in Figure 7. The oxygen fraction in film increases with the oxygen flow rate, which confirms the above-mentioned absorption of excess oxygen. Similar consequence has also been found in the dual ion beam sputter deposition of $\mathrm{ZnO}$ [21].

The electrical resistivity, the carrier mobility, and the carrier concentration of each MZO-coated PES prepared at different oxygen flow rates are shown in Figure 8. The MZO deposited without additional oxygen shows the lowest resistivity of $8.32 \times 10^{-3} \Omega \mathrm{cm}$. The resistivity increases with the oxygen flow rate. The same trend has also been reported in ion beam sputter deposition of ITO films [25] and facing targets sputtering deposition of aluminum-doped zinc oxide (AZO) films. It could be due to both the carrier mobility and the carrier concentration decreasing with the oxygen flow rate. According to results of MZO crystallinity, the increased grain and subgrain boundaries might be the main reason to retard the carrier mobility in films deposited with additional oxygen flow [26]. The lesser oxygen vacancy and $\mathrm{Mo}^{6+}$ in a relatively stoichiometric film deposited with oxygen flow might be the reason reducing the carrier concentration.

The transmittance spectra of MZO-coated PES prepared at different oxygen flow rates are shown in Figure 9(a). The specimens deposited without additional oxygen flow present an average transmittance of $81.3 \%$ for visible region and an absorption edge at shorter wavelength. Other specimens deposited with introducing oxygen flow show slightly lower average transmittance values between 79.2 to $80 \%$. It could be due to the scattering by increased internal boundaries [27]. The optical absorption edge in near-ultraviolet region also shifts toward visible region with the oxygen content. The estimated optical band gap shown in Figure 9(b) clearly presents a same tendency, decreasing from 3.46 to $3.20 \mathrm{eV}$. It corresponds to the decrease of carrier concentration as shown in Figure 8. According to the Moss-Burstein bandfilling effect [28], for a semiconductor with excess carriers, its conduction band or valence band will be filled with some carriers, which make the following carriers require higher energy to excite. It explains that the absorption edge of TCO shifts to longer wavelength (lower energy) with decreasing carrier concentration [29]. Based on the results of transmittance and resistivity, the calculated FOM as shown in Figure 10 also decreases with the deposition oxygen flow rate. The MZO-coated PES deposited without introducing oxygen flow exhibits the highest FOM of $67.3 \times 10^{-5} \Omega^{-1}$.

\section{Conclusions}

In this study, a molybdenum-doped zinc oxide (MZO) was deposited onto a flexible polyethersulfone (PES) substrate 
by using a dual ion beam sputtering system. The influences of oxygen flow rate of the deposition on the acquired MZO films have been investigated. It is found that the film deposited in the atmosphere without introducing oxygen exhibits the best optical transmittance of $82.9 \%$ at $550 \mathrm{~nm}$ wavelength and electrical resistivity of $8.32 \times 10^{-3} \Omega \mathrm{cm}$. However, both the optical transmittance and electrical resistivity of films increase with the introduced oxygen flow rate. The additional oxygen flow increases the oxygen fraction and causes a more stoichiometric composition in films, which reduces the oxygen vacancy and the free carrier concentration. The addition of oxygen also promotes the growth of grains and projections in MZO films. These increased boundaries not only reduce the carrier mobility but also cause scattering loss of transmittance for visible light. The decreases of both carrier concentration and carrier mobility result in the lower transparency and conductivity of MZO films deposited with introducing oxygen flow.

\section{References}

[1] M. Higgins, S. Uekusa, P. Nakano, and K. Yokogawa, "Postdeposition annealing influence on sputtered indium tin oxide film characteristics," Japanese Journal of Applied Physics, vol. 33, no. 1, pp. 302-306, 1994.

[2] A. Subrahmanyam, C. Suresh Kumar, and K. Muthu Karuppasamy, "A note on fast protonic solid state electrochromic device: NiOx/Ta2O5/WO3-x," Solar Energy Materials and Solar Cells, vol. 91, no. 1, pp. 62-66, 2007.

[3] X. Jiang, F. L. Wong, M. K. Fung, and S. T. Lee, "Aluminumdoped zinc oxide films as transparent conductive electrode for organic light-emitting devices," Applied Physics Letters, vol. 83, no. 9 , pp. 1875-1877, 2003.

[4] A. Gupta and A. D. Compaan, "All-sputtered 14\% CdS/CdTe thin-film solar cell with $\mathrm{ZnO}$ : Al transparent conducting oxide," Applied Physics Letters, vol. 85, no. 4, pp. 684-686, 2004.

[5] M. J. Alam and D. C. Cameron, "Optical and electrical properties of transparent conductive ITO thin films deposited by sol-gel process," Thin Solid Films, vol. 377-378, pp. 455$459,2000$.

[6] K. Ellmer, "Magnetron sputtering of transparent conductive zinc oxide: relation between the sputtering parameters and the electronic properties," Journal of Physics D, vol. 33, no. 4, pp. R17-R32, 2000.

[7] S. Kim, W. Lee, and C. Lee, "Influence of $\mathrm{ZnO}$ buffer layer thickness on electrical and optical properties of GZO thin films deposited on polymer substrates," Materials Science and Technology, vol. 23, no. 3, pp. 303-306, 2007.

[8] D. S. Ginley and C. Bright, "Transparent conducting oxides," MRS Bulletin, vol. 25, no. 8, pp. 15-21, 2000.

[9] S. M. Kim, Y. S. Rim, M. J. Keum, and K. H. Kim, "Study on the electrical and optical properties of ITO and AZO thin film by oxygen gas flow rate," Journal of Electroceramics, vol. 23, no. 2-4, pp. 341-345, 2009.

[10] F. O. Adurodija, H. Izumi, T. Ishihara, H. Yoshioka, M. Motoyama, and K. Murai, "Effect of laser irradiation on the properties of indium tin oxide films deposited by pulsed laser deposition," Applied Surface Science, vol. 177, no. 1-2, pp. 114121, 2001.

[11] V. Sittinger, F. Ruske, W. Werner, C. Jacobs, B. Szyszka, and D. J. Christie, "High power pulsed magnetron sputtering of transparent conducting oxides," Thin Solid Films, vol. 516, no. 17, pp. 5847-5859, 2008.

[12] C. C. Kuo, C. C. Liu, C. C. Lin, Y. Y. Liou, J. L. He, and F. S. Chen, "Effects of oxygen gas flow rate and ion beam plasma conditions on the opto-electronic properties of indium molybdenum oxide films fabricated by ion beam-assisted evaporation," Thin Solid Films, vol. 516, no. 16, pp. 5612-5617, 2008.

[13] Y. S. Choe, J. H. Chung, D. S. Kim, and H. K. Baik, "Ion beam sputtering of $\mathrm{SnO}_{2}$ with low energy oxygen ion beams," Thin Solid Films, vol. 341, no. 1, pp. 230-233, 1999.

[14] D. F. Lii, J. L. Huang, I. J. Jen, S. S. Lin, and P. Sajgalik, "Effects of annealing on the properties of indium-tin oxide films prepared by ion beam sputtering," Surface and Coatings Technology, vol. 192, no. 1, pp. 106-111, 2005.

[15] Y. Suzuki, T. Yotsuya, M. Yoshitake, K. Takiguchi, and S. Ogawa, "Modification of $\mathrm{ZnO}$ crystal orientation in dual ion beam sputtering deposition," Nuclear Inst. and Methods in Physics Research B, vol. 39, no. 1-4, pp. 732-735, 1989.

[16] G. Haacke, "New figure of merit for transparent conductors," Journal of Applied Physics, vol. 47, no. 9, pp. 4086-4089, 1976.

[17] Y. Qu, T. A. Gessert, T. J. Coutts, and R. Noufi, "Study of ion beam sputtered $\mathrm{ZnO}$ films as a function of deposition temperature," Journal of Vacuum Science and Technology A, vol. 12, no. 4, pp. 1507-1512, 1994.

[18] T. Tsuji and M. Hirohashi, "Influence of oxygen partial pressure on transparency and conductivity of RF sputtered Aldoped $\mathrm{ZnO}$ thin films," Applied Surface Science, vol. 157, no. 1, pp. 47-51, 2000.

[19] W. F. Wu and B. S. Chiou, "Deposition of indium tin oxide films on polycarbonate substrates by radio-frequency magnetron sputtering," Thin Solid Films, vol. 298, no. 1-2, pp. 221-227, 1997.

[20] J. S. Cho, K. H. Yoon, and S. K. Koh, "Microstructure of indium oxide films in oxygen ion-assisted deposition," Thin Solid Films, vol. 368, no. 1, pp. 111-115, 2000.

[21] F. Quaranta, A. Valentini, F. R. Rizzi, and G. Casamassima, "Dual-ion-beam sputter deposition of $\mathrm{ZnO}$ films," Journal of Applied Physics, vol. 74, no. 1, pp. 244-248, 1993.

[22] M. Chen, Z. L. Pei, C. Sun, L. S. Wen, and X. Wang, "Formation of $\mathrm{Al}$-doped $\mathrm{ZnO}$ films by dc magnetron reactive sputtering," Materials Letters, vol. 48, no. 3-4, pp. 194-198, 2001.

[23] Y. Meng, X. L. Yang, H. X. Chen et al., "A new transparent conductive thin film $\operatorname{In}_{2} \mathrm{O}_{3}$ :Mo," Thin Solid Films, vol. 394, no. 1-2, pp. 219-223, 2001.

[24] C. V. Ramana, V. V. Atuchin, V. G. Kesler et al., "Growth and surface characterization of sputter-deposited molybdenum oxide thin films," Applied Surface Science, vol. 253, no. 12, pp. 5368-5374, 2007.

[25] D. Kim, Y. Han, J.-S. Cho, and S.-K. Koh, "Low temperature deposition of ITO thin films by ion beam sputtering," Thin Solid Films, vol. 377-378, pp. 81-86, 2000.

[26] R. Abbaschian, L. Abbaschian, and R. E. Reed-Hill, Physical Metallurgy Principles, PWS publishing Company, Boston, Mass, USA; Cengage Learning, Stanford, Calif, USA, 4th edition, 2008.

[27] Y. Hu, X. Diao, C. Wang, W. Hao, and T. Wang, "Effects of heat treatment on properties of ITO films prepared by rf magnetron sputtering," Vacuum, vol. 75, no. 2, pp. 183-188, 2004.

[28] E. Burstein, "Anomalous optical absorption limit in InSb," Physical Review, vol. 93, no. 3, pp. 632-633, 1954. 
[29] T. Umebayashi, T. Yamaki, H. Itoh, and K. Asai, "Band gap narrowing of titanium dioxide by sulfur doping," Applied Physics Letters, vol. 81, no. 3, pp. 454-456, 2002. 

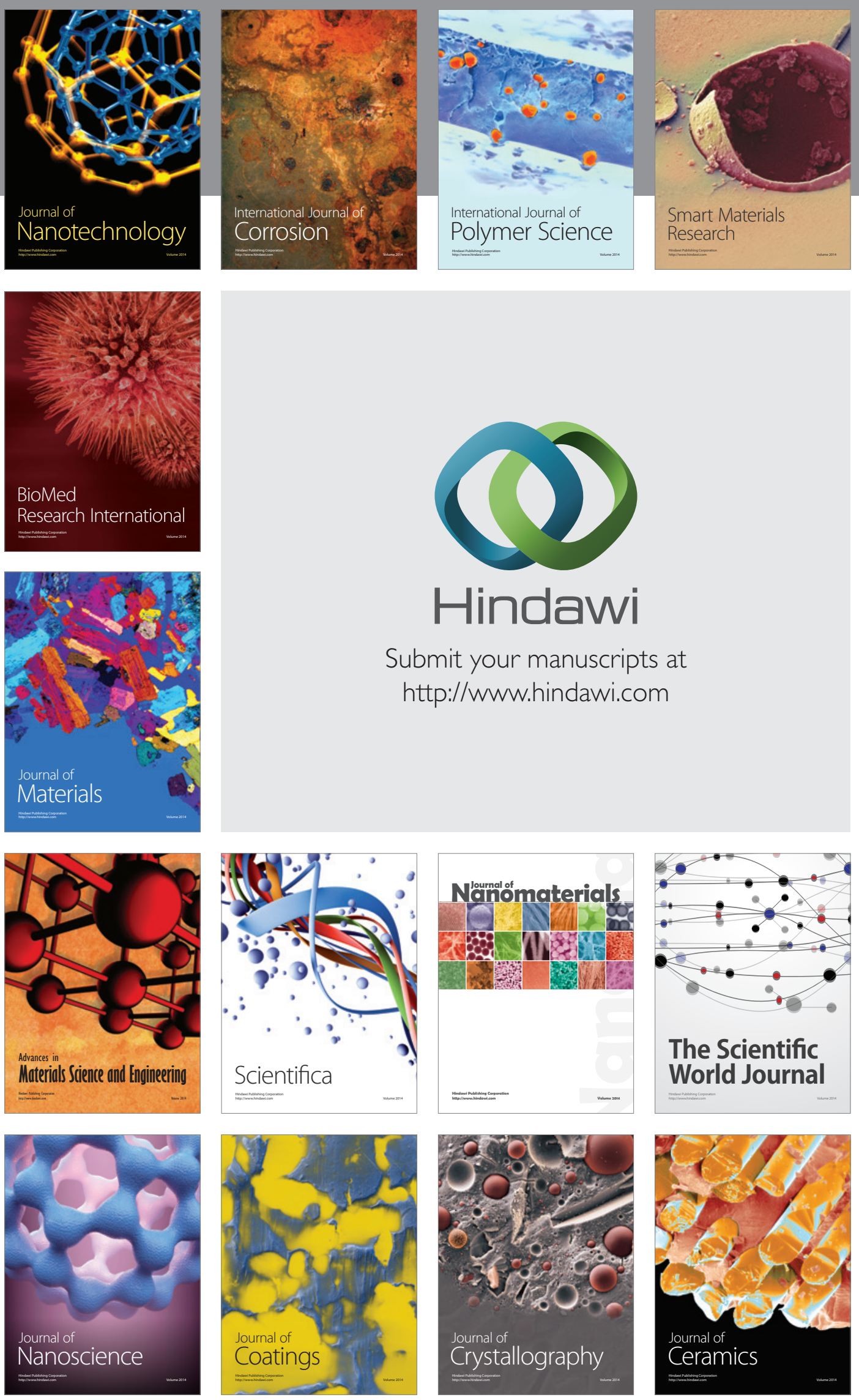

The Scientific World Journal

Submit your manuscripts at

http://www.hindawi.com

\section{World Journal}

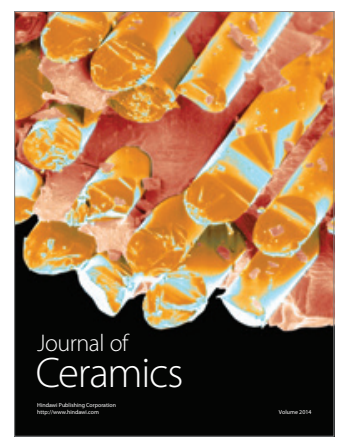

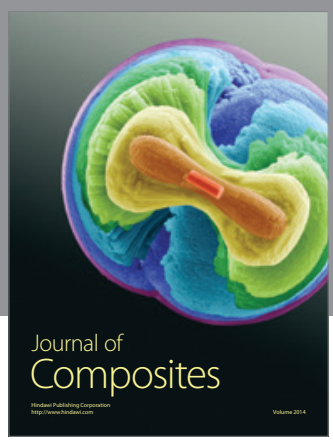
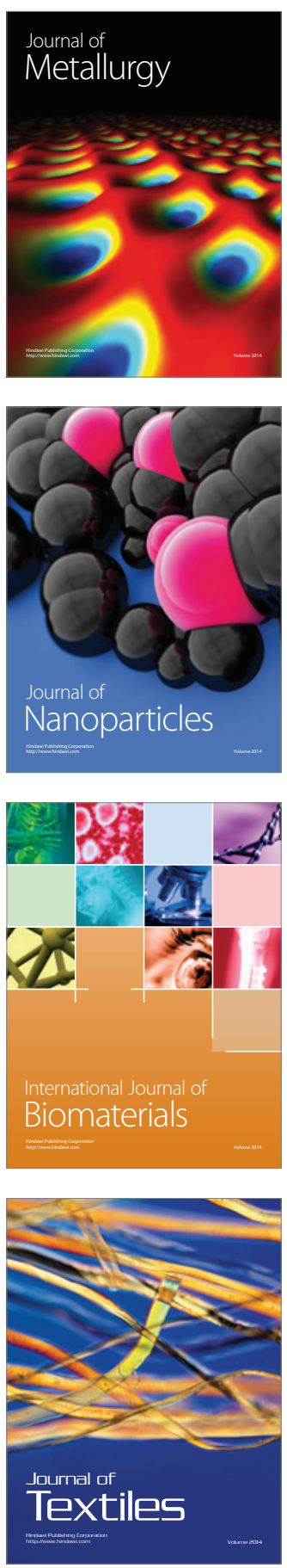